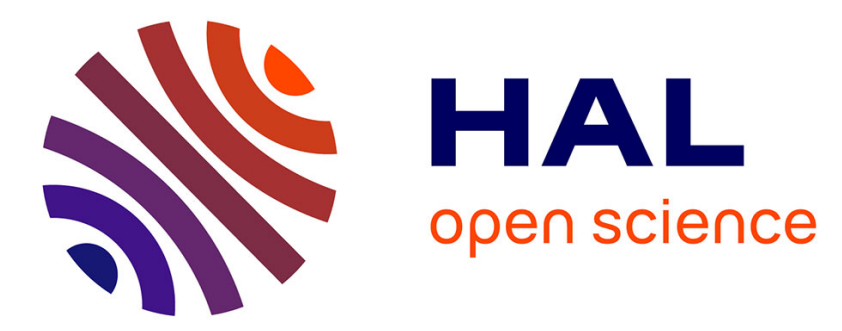

\title{
Use cases for social data analysis with URREF criteria
}

Claire Laudy, Valentina Dragos

\section{To cite this version:}

Claire Laudy, Valentina Dragos. Use cases for social data analysis with URREF criteria. 2020 IEEE 23rd International Conference on Information Fusion (FUSION), Jul 2020, Rustenburg, South Africa. pp.1-8, 10.23919/FUSION45008.2020.9190619 . hal-03217147

\section{HAL Id: hal-03217147 https://hal.science/hal-03217147}

Submitted on 4 May 2021

HAL is a multi-disciplinary open access archive for the deposit and dissemination of scientific research documents, whether they are published or not. The documents may come from teaching and research institutions in France or abroad, or from public or private research centers.
L'archive ouverte pluridisciplinaire HAL, est destinée au dépôt et à la diffusion de documents scientifiques de niveau recherche, publiés ou non, émanant des établissements d'enseignement et de recherche français ou étrangers, des laboratoires publics ou privés. 


\section{Use cases for social data analysis with URREF criteria}

\author{
Claire Laudy \\ Thales TRT \\ Palaiseau, France \\ claire.laudy@ thalesgroup.com
}

\author{
Valentina Dragos \\ ONERA - The French Aerospace Lab \\ Palaiseau, France \\ valentina.dragos@onera.fr
}

\begin{abstract}
Social data analysis has gained prominence in a wide range of domains as it provides users with the opportunity to communicate and share posts and topics. Automated analysis and reasoning about such data potentially derive meaningful insights, with tremendous potential for applications. However, the sheer volume, noise, and high dynamics of social data impose challenges that hinder the efficacy of algorithms. Automated approaches and classification models require then significant resources to be developed and prove to be often relevant to only a limited number of tasks. Imperfections of inputs, precision of techniques and accuracy of results need to be accounted and assessed as the process runs. This paper discuses two use cases allowing the investigation of implicit and explicit uncertainty arising when processing data gleaned on social media. The objective of this paper is twofold. The first objective is to set up the ETUR use case on social media analysis by adopting two tasks on opinion mining for cyberspace surveillance and information extraction for crisis analysis, respectively. The second objective is to discuss an overall methodology allowing the identification and assessment of uncertainties underlying each task The paper introduces two illustrations of social data analysis, investigates various sources of uncertainty and describes a methodology to select criteria for uncertainty assessment.
\end{abstract}

Index Terms-Social media analysis, information extraction, opinion mining, uncertainty

\section{INTRODUCTION}

The Internet is currently an essential channel for organizations and users promoting a diversity of topics, from presidential elections to consumer concerns, environmental issues or societal debate [1]. At the other end of the chain, it is also a major source of information for organizations and citizens to be informed on various situations.

The lack of social accountability in many digital platforms in the cyber-physical space [2] yields plenty of incentives for unprecedented forms of misuse. Disinformation [3], [4] propaganda [5] or social unrest [3] are just a few examples of ill-uses lurking in a technology largely accessible. Social stream is the collected information from social networks that shows how users share, view or engage with online content or profiles. Social stream analysis can provide a new slant on security applications and can lead to important insights that the use of analytics did not explore.

This paper aims to sample the state of the art in using social media analytics for security applications from a methodological perspective and has a twofold contribution. First, we provide an overview of main challenges of social streams exploration and discuss methods developed to overcome those challenges. Secondly, we present two practical applications of social media exploitation for intelligence analysis and crisis management.

Through the presentation of applications, we aim at illustrating two different uses of Internet and more specifically the social media, within two different contexts of use and user needs. The first application shows how social media can support intelligence in the context of wide information gathering, from unfiltered sources of information. The aim is to detect situations or events of interest that would require further follow-up and monitoring. The approach uses statistical machine learning techniques. The second application is useful when such situations of interest have been detected and have to be monitored. This application relies on a model-based approach and it is useful for use cases where information sources that are relevant to monitor the situation have been selected.

The main goal of the paper is to provide insights in order to understand current capabilities for social streams analysis for security applications and to set two use cases for social data analysis for the ETUR (Evaluation of Techniques for Uncertainty Representation ${ }^{1}$ ) community.

The rest of this paper is organized as follows: section II discusses a selection of methods and techniques developed for social data analysis. Section III describes the first use case, intended to mine opinions from social streams; the second case addresses the exploitation of social media for crisis management and is discussed in section IV. Section V introduces the methodology adopted to analyze the uncertainty in the light of URREF (Uncertainty Representation and Reasoning Evaluation Framework) criteria. Section VI concludes this paper.

\section{RELATED WORK}

Social data analysis has been actively studied in the past few years, in an effort to both investigate and understand online content. Solutions were developed for sentiment analysis and opinion detection, although those terms are not accurately defined and are sometimes interchangeable. More recent applications are in the field of security, with emphasis on extremist content detection [6] and understanding online propaganda [7].

\footnotetext{
${ }^{1}$ https://eturwg.c4i.gmu.edu/
} 
From a practical standpoint, applications aim at: hate speech [8] and violence detection [9], extremist content analysis [10], exploration of online communities [11] and online contagion mechanisms [5], detection of opinion leaders [12], and analysis of behavioural patterns [13].

Classification algorithms were widely used for sentiment analysis [14], and those techniques mostly depend on feature engineering and manually defined rules and resources, such as dependency and causality relations, n-grams or lexicons. They leverage the bag-of-word representations and convent the corpus into a term-document matrix, following the largely adopted routine of normalization and stemming.

More recently, the so called end-to-end approaches based on neural network techniques without feature engineering became popular for social data analysis [15]. Those solutions rely upon embedded structures, such as low dimensional word vectors which contain shallow semantic information. Similar approaches are developed by adopting supervised sequence labelling. Thus, Hidden Markov models and conditional random fields are used by Chen and colleagues to extract aspect and polarity from social data [16]. To alleviate the need to use large amounts of labelled data sets for training purposes, unsupervised methods based on topic modeling [17] or ontologies [18] were also developed.

Although supervised solutions achieve remarkable accuracy for sentiment analysis, robustness in processing a mix of multidomain data [19] and the use of manually tagged inputs have been plaguing the adoptability of those approaches.

From a different perspective, a variety or semantic resources [20] have been built to capture and model concepts describing subjective engagement, polarity and other attributes specific to opinion detection and sentiment analysis. Based on those resources, techniques were developed which are able to detect instances of concepts within online content and to emphasize on words that are relevant to aspect, emotions and opinions [21]. Many of those resources come with limitations as they are designed to achieve broad coverage and fail to capture domain-specific standpoints [22].

However, most work in social stream analysis focuses on overall opinion detection or sentiment analysis, regardless of the entities, targets or topics mentioned in the content. The algorithms detect sentiments or opinion by assuming a known target. Taking a step further, recent studies by Schoene and de Mel [23] investigate the correlation of topics and emotions while Vijayaraghavan and colleagues addressed the classification of topics along with the sentiment [24]. Following a similar research line, Schmitt et al. employed an end-toend trainable neural network to detect aspects and classify polarities jointly.

Social data analysis is currently a challenging task for solutions based on rule-based, statistical methods, learning or natural language processing, and uncertainty affects the results of those techniques. This paper tackles the analysis of uncertainty for social data processing with URREF criteria and introduces two use cases for social stream analysis. The paper investigates the nature of uncertainty affecting those use cases by identifying the sources of uncertainty and relating them to uncertainty criteria defined by the URREF ontology.

\section{USE CASE: OPINION DETECTION IN SOCIAL STREAMS}

\section{A. Goal and application context}

This use case aims at detecting opinions in social media. The approach is grounded in the cognitive foundations of the appraisal theory, developed by White and Martin [25]. The appraisal theory structures appraisal expressions under three main basic categories describing attitudes, engagement and graduation, as shown in fig. 1 .

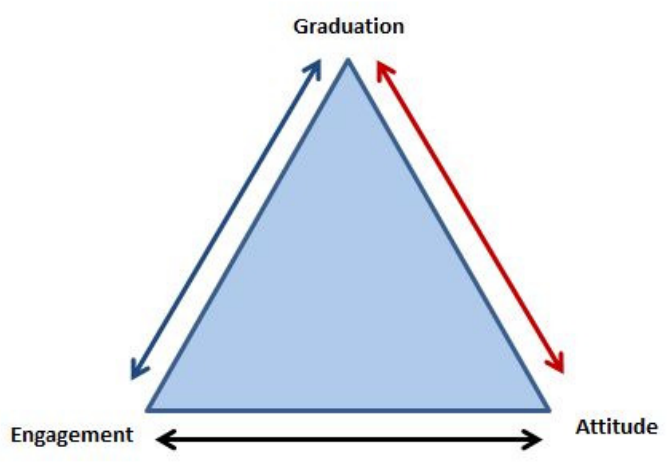

Fig. 1. Appraisal categories

The frame also highlights the mechanisms by which text convey positive and negative attitudes, as shown in tab. I.

TABLE I

APPRAISAL CATEGORIES SPLIT INTO POLARITY

\begin{tabular}{|l|l|l|l|}
\hline Polarity & Appreciation & Engagement & Affect \\
\hline Positive & Amazing & Obviously & Joy, happy \\
\hline Negative & Awful & Apparently & Miserable \\
\hline
\end{tabular}

Starting with the three systems introduced by the appraisal theory, a semantic resource was build modeling finer categories under each system, and highlighting terms and expressions specific to each category. This semantic-driven approach goes beyond the generally accepted definitions of sentiment and opinions, and focuses on appraisal expressions to describe the way humans express their attitudes, appreciations and engagements.

The application context of this use-case is a cyberspace exploration task designed to support defence and homeland security intelligence practitioners in their efforts to gather data in order to understand emergent phenomena, such as online hate proliferation or online propaganda. Linguistic clues of appraisal categories are used as indicators of subjective content to be collected and further analysed in order to understand the way online users express their extreme attitudes, embrace or support extreme ideologies and ideas. 


\section{B. General approach}

The general approach relies on semantic annotation augmented with processing methods capable to perform a quantitative analysis of data gleaned on social media. The approach consists of several phases implemented to gather, process and analyse social data, see fig. 2.

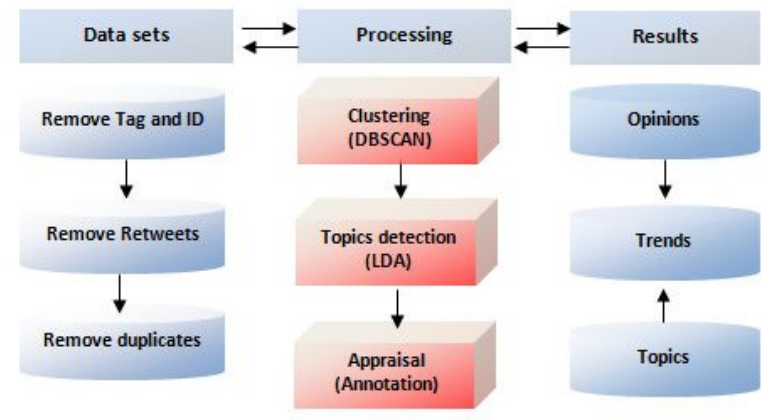

Fig. 2. Hybrid architecture opinion detection

- Data acquisition: is done by crawling several sets of tweets with specific keywords and additional constraints to select only posts written in English. This phase was carried out as a straightforward step and relies only on data content of both keywords and posts and the ability of API used to mine the social network.

- Data preprocessing and cleaning: prior to conducting experiments, tweets are broken down into sentences and then the preprocessing includes: tokenization, lower casing all tokens, removing images, punctuation and stop words. Furthermore usernames, retweets, duplicates and web references are removed as well. The rationale behind this cleaning step is that tweets commonly get large number of retweets and keeping them in the dataset will produce large clusters containing redundant tweets rather than tweets with similar features. This will consequently reinforce false patterns and increase run time.

- Data processing: unsupervised classification is used to classify data and then topics are detect within each cluster as to pool tweets into more homogeneous groups and show which topics are the most dominant within each cluster;

- Semantic annotation: detects instances of concepts within tweets and attaches them as additional information to various text paragraphs.

- Data analysis: a trend-detection strategy is adopted based on appraisal categories to infer the polarity of categories detected in topics and to aggregate trends at topic level from appraisals detected at tweet level.

The appraisal ontology is represented in OWL [26] and is composed of 46 concepts structured on a 6-levels hierarchy; the model also has 4 ObjectProperties and 2 DataType propertie, 50 nodes, 75 edges and 268 instances of concepts. Tweets associated to each topic were annotated through the ontology of appraisals and the result is a number of basic appraisal categories associated to each tweet.

\section{USE CASE: SEMANTIC INFORMATION FUSION FOR CRISIS MONITORING}

\section{A. Goal and application context}

The application presented in this use case focuses on the monitoring of a specific situation of interest. It relies on open and operational sources of information. Operational sources enable to build the knowledge model and provide access to restricted information sources. Crisis throughout the world recently prompted new attention to the role that social media platforms can play in emergency situations and response efforts. The open sources, mainly social media and encyclopedic information sources such as Wikipedia, provide complementary points of view on the monitored situation. When an emergency situation is on-going, the PSO (Public Safety Organizations) officers pile up a large amount of information in order to have a better understanding of the ongoing situation and evaluate the level of emergency and severity. These information items may be received either by other PSOs, or gathered on social media, where citizens involved or witnesses of the situation provide descriptions and comments.

One of the issues for using such an amount of information is to be able to access relevant parts of it efficiently. For example, this enables highlighting schemes of emergency events for instance.

\section{B. General approach}

The Semantic Information Fusion application relies on the use of semantic graph structures to store information and uses a graph algorithm to carry out the fusion process [27]. It enables three different operations on networks of information:

- Report synthesis: The emergency messages related to the same emergency situation are processed through the SIF module, so to provide the PSOs with a synthetic and nonredundant description of the situation. The event descriptions are fused by giving the most precise description that contains all the elements of the initial messages. The type of event reported is the most generic common subtype of all the initial reports, and the content of the synthetic report contains all the information contained in the initial reports. Whenever a piece of information is redundant from one report to another, the fusion function eliminates this redundancy. To illustrate this function, let us consider the following messages that could be collected in an emergency center.

T1: There are lots of cars stuck in a tunnel due to snow. T2: There might be a crash inside the tunnel.

T1 will be detected by typing service of type CarEvent and $\mathrm{T} 2$ of type Accident. Therefore, the synthesis of T1 and $\mathrm{T} 2$ will generate an event report of type CarAccident. Furthermore, the synthetic report will contain both sentences of $\mathrm{T} 1$ and $\mathrm{T} 2$.

- Information query: All the instances of information corresponding to a specified graph pattern may be found within 
a network of information, through the information query function. PSO will look for instance for all instances of events of type fire in a habitation building during a weekend that are listed in their data bases.

- Information evaluation: The function provided by the Information Evaluation service is the evaluation of the degree of certainty one may grant an event, given all the information available on this event. This evaluation relies on the use of a network or database of event and/or testimony descriptions. The testimonies and descriptions are scored according to the degree of confidence the PSO officer has in this information. The main function provided by the Information Evaluation module, is the query for degree of certainty that a specific situation or event is on-going. When the PSO wants to evaluate the level of certainty that an event occurred or is ongoing, they query the available information items on this event. Given the degree of certainty associated with each information item, a global evaluation of the veracity of the event is processed. The information evaluation approach relies on an extension of the Transferable Belief Models to graph structures (see [28]).

In addition to those operations, event typing is carried out in order to manage emergency messages and it relies on the deep analysis of the messages received by the PSOs. The first step of this deep analysis is to type the emergency descriptions (i.e. the messages), according to the type of emergency they describe. The possible emergency types are defined in the domain ontology provided to the fusion module.

TABLE II

EXAMPLE OF EVENT TYPES, KEYWORDS DATASET PROVIDED TO THE IMS AS PARAMETERIZATION

\begin{tabular}{|l|l|}
\hline Accident & Accident, crash, omnettomuus \\
\hline CarEvent & Car, truck, road \\
\hline TrainEvent & Train \\
\hline Fire & Fire, palokunta, incendie \\
\hline Flood & Flood, innondation \\
\hline BusEvent & Bus \\
\hline
\end{tabular}

To type the emergency messages, we use a data set of keywords associated to one or more emergency types, see tab. II. As keywords are detected in a message, all possible emergency types are selected. The type finally associated to the emergency is the most general common subtype, according to the domain ontology (see image 3 for an example).

In order to manage emergency messages, IE/SIF relies on the deep analysis of the messages received by the PSOs. The first step of this deep analysis is to type the emergency descriptions (i.e. the messages), according to the type of emergency they describe. The possible emergency types are defined in the domain ontology provided to the fusion module.

For example, the following messages will be typed as follows:

Sentence: There are lots of cars stuck in a tunnel due to snow. There might be a crash inside the tunnel.

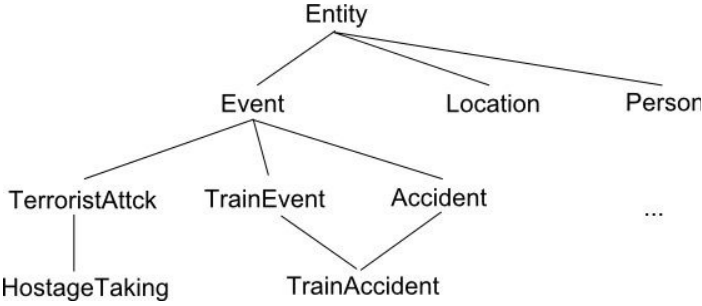

Fig. 3. Example of a type hierarchy

\section{Potential event types : [CarEvent, Crash, Accident] Selected event type : CarAccident}

Remarks: those use cases for social data analysis are complementary with respect to several standpoints: first, crisis management requires fast and accurate procedures to manage real-life events; low-quality sources or inaccurate processing have an important impact on the quality of results. Ideally, the use case relies upon a reduced number of highly reliable sources to feed the processing chain. Opinion detection involves a rather long-time analysis of how user's orientations (political, religious, etc.) changes in time. The overall approach relies upon an important number of items gleaned online although the overall approach is less impacted by the integration of partially or completely unreliable sources, since having a large volume of data should cope the inaccuracy of some items.

How the uncertainty affects both use-cases is discussed hereafter.

\section{Methodology FOR UNCERTAINTY ANALYSIS AND} ASSESSMENT

The methodology adopted for uncertainty analysis has three phases, namely:

Phase 1: Perform an analysis of the nature of social data, in order to identify key factors contributing to success and failure of applications exploiting this type of data.

Phase 2: Identify critical processing steps that are specific to use cases and can induce additional uncertainty to affect the outcome;

Phase 3: Identify the set of URREF criteria over which the use cases previously introduced are to be analysed. The set of criteria are selected in such a way that they will are relevant for social data, and have, potentially the ability to cover all uncertainty sources identified for both use cases.

\section{A. Challenges of social data analysis}

Analysis of online streams comes with challenges stemming from both the specific nature of data created on digital platforms and the difficulties of social media exploration, as discussed hereafter.

Specificity of data: data collected on social media data are vast, noisy, unstructured, inherently dynamic and heterogeneous in nature. Moreover, they convey reports on real-life facts and events augmented with personal points of view, such as evaluations, attitudes, and emotions. Therefore, social data 
analysis is challenging for traditional data mining approaches that are often too slow and expensive, rely on sample sizes, and come with biases leading to errors.

Limitation of access and impact of secondary sources: An important volume of online data is released on DarkWeb [29], a problematic side of Web made of encrypted portions of the Internet that are not indexed by search engines and thus cannot be listed on results pages returned by search engines to user queries. Collection, processing and sharing of such content require specific procedures to be set up or the use of secondary sources. Nevertheless, secondary sources can introduces biases, or even truncate or alter the original set.

Influence of platforms and media-induced bias: $\mathrm{Cy}$ berspace is an artificial, man-made environment, with data and interactions framed in a particular manner. This is the main reason any social media platform induces bias in how information is viewed by observers. Moreover, those observers can either be a part of the platform when undertaking their analysis or adopting a more direct approach to collect data via technical procedures. Those procedures are built on application programming interfaces (API) which are subroutines provided by social platforms to access their collections of data, which are also stored in proprietary formats, most of the time. This is a major drawback, as the collection requires an effort to translate data into formats easier to process and has additional limitations. As an example, although it is possible to extract data directly from Twitter archives via an request using external calls to the Twitter API, the volume accessed during one session cannot exceed 3200 tweets.

Security and ethical constraints, privacy protection: There are significant security related and ethical constraints to obtain first-hand information about sites, portals or content on social media platforms created by terrorists or extremist groups, from intelligences services, for example. Regarding privacy, data gathering and remote analysis for research purposes requires procedures and techniques that should be employed lawfully, as to make sure the overall process stay within the law.

In additional to those general challenges, social media exploration is also affected by technical bottlenecks, such as: multilingual issues, multimodality content, relevance and coherence of data sets, contextual information, aggregation and correlation of items, etc. Outcomes are then affected by uncertainty, and the URREF ontology offers a basis to account and assess those uncertainties, as discussed hereafter.

\section{B. URREF ontology and sources of uncertainty}

The URREF ontology was developed within the ISIF's Evaluation of Techniques for Uncertainty Representation Working Group (ETURWG) in order to asses different aspects of uncertainty in information fusion systems.

The framework considers uncertainty representation and reasoning components of fusion systems as subjects under evaluation [30], but the model also defines criteria for secondary evaluation subjects such as sources of information, piece of information and fusion methods.
URREF criteria have generic definitions and can be instantiated for applications with coarse or finer granularity levels: evaluation metrics can be defined for data analysis [31], or more particularity for data specific types [32] or attributes: reliability and credibility [33], trust and self-confidence [34] or veracity [35]. While allowing a continuous analysis of uncertainty representation, quantification and evaluation [36], URREF criteria are detailed enough to capture model-embedded uncertainties [37], their propagation in the context of the decision loop [38] and offer a basis to compare different fusion methods [39]. URREF criteria were used for uncertainty tracking and investigation for several applications: activity detection for rhino poaching [40] and imagery for large area protection [41].

Social data analysis for crisis management or opinion detection is affected by uncertainty induced by online data gathering and the integration of results provided by several algorithms. For both use cases, the process under analysis is the overall approach adopted to analyze social data.

For the use cases considered in this paper, uncertainty enters in three main forms: uncertainty of input data, uncertainty of data enrichment procedures, and processing uncertainty encompassing uncertainty of specific algorithms, as shown in tab. III.

TABLE III

SOURCES OF UNCERTAINTY

\begin{tabular}{|l|l|l|}
\hline Sources of uncertainty & Use case 1 & Use case 2 \\
\hline Data sets & Data collected & Data selected \\
\hline Data enrichment & Annotation of data & Evaluation of information \\
\hline Data processing & Detection of topics & Synthesis of reports \\
\hline
\end{tabular}

Additionally, data handling from collection of data sets to interpretation of results is also prone to uncertainties.

- Data collection from social media

- Data refinement or enrichment

- Data processing or fusion

- Data handling throughout the process

First, both use cases are affected by the quality of input data, although items are selected for one use case and automatically gleaned with keywords for another. Then, data refinement and processing are implemented with specific procedures. The next section discusses the selection of URREF criteria capturing those uncertainties.

\section{URREF criteria for uncertainty analysis}

Uncertainty of input data: The criterion discussed in this section refers to the characterisation of data items collected on the Internet before they enter any processing. For both use case the relevant criterion for uncertainty analysis is relevance to problem. Relevance to problem highlights a piece of information that is specific to applications or tasks. Intuitively, relevance to problem is impacted by the ability of selecting data sources that are related to the case.

For crisis management, relevance to problem can be related to spatial and temporal coordinates of online sources, as to 
make sure that the item provided is related to the crisis under analysis at least in the light of time and space, regardless of its content. For opinion detection, gathering data with keywords is intended to bring into analysis a set of relevant items.

However, both cases are impacted by challenges of social data processing: for crisis monitoring, time zone and geographical localisation prove to be sometimes unreliable for online sources. For the opinion detection use case, key words are provided to API of platforms that will eventually gather data, although there is no control over their internal procedures.

Uncertainty of data enrichment : Data enrichment creates, for both use cases, a meta description of initial data sets, by attaching an estimation of the quality, in the case of crisis monitoring and an annotation with appraisal categories for opinion detection use case.

Two criteria can be used to capture induced uncertainties, both under the umbrella of uncertain evidence: credibility and ambiguity.

Uncertainties of data annotation are captured by ambiguity, an URREF criterion intended to capture the ability for an information item to equally refer to several different entities. As annotation is performed automatically in the light of the appraisal ontology, the same item can be annotated with contradictory concepts.

As data used for information evaluation are more complex items, they can be characterised by credibility. Under URREF, credibility is specified by three sub-criteria : observational sensitivity, selfConfidence and objectivity, intended to capture the ability of an item to be credible or worthy of trust. For second use case, selfConfidence is relevant to analyse the quality of data items, although credibility itself can be also be taken into consideration.

Uncertainty of data processing: Data processing is implemented in order to detect topics or to combine information items into more synthetic reports.

Two mechanisms induce uncertainties when performing this processing: first, identification of topics is based on classification with unsupervised approaches, since those methods build classes without needing data annotation beforehand. However, the homogeneity of classes and their capacity to cluster all items related to the same item is questionable. Second, synthesis of information reports is carried out by fusing information items, which can be correlated, inconsistent or of different quality.

Under URREF ontology, consistency is the criteria able to capture uncertainties of topics detection. and the criterion can be estimated in the form of a homogeneity coefficient, capturing how coherent the classes created by clustering algorithms are.

To capture the uncertainty of report synthesis, incompleteness and dissonance are the two criteria selected. First, incompleteness can highlight data items conveying specific features. This can be the case with sources which specify the type of entities (bus, vehicle, or person) without realizing that they are part of a group (convoy). Although they provide reliable attributes, those sources ignore the overall picture and thus the evidence is not complete. Such incomplete evidence is inherent when using natural language data. Then, dissonance is a criterion relevant when various items are integrated to build a synthetic report. This criterion implies a clash between features identified within different items and it can also be captured in the form of violations of ontological assertions.

Uncertainty of data handling: Traceability and interpretation are two criteria provided by URREF to analyse uncertainty related to data handling. For use cases discussed in this paper, interpretation can be used as related to the number and quality of topics that the detection method is able to identify given a collection of data. The second data-handling criterion, traceability captures the ability of keeping track of the evolution of the final situation, as additional items are added, and therefore to chronologically intercorrelate various reports in a way that is verifiable. Traceability is relevant only for the second use case, as it builds situation to monitor crisis, and items integrated can be crucial for the course of action. We do not consider traceability for the first use case as the method integrates a large amount of items, although individually each item is of limited influence on the final outcome.

Comparison of uncertainty analysis: An overall view of uncertainty criteria selected for both use cases is shown in tab. IV.

TABLE IV

UNCERTAINTY CRITERIA FOR USE CASES

\begin{tabular}{|l|l|l|}
\hline Criteria & Use case 1 & Use case 2 \\
\hline Data & Relevance to Problem & Relevance to Problem \\
\hline Enrichment & Ambiguity & SelfConficence, Credibility \\
\hline Processing & Consistency & Dissonance, Incompleteness \\
\hline Data handling & Interpretation & Traceability \\
\hline
\end{tabular}

Besides showing criteria specific to each use case according to input data and processing chains, the image fig. 4 also shows how those criteria are to be considered in time.

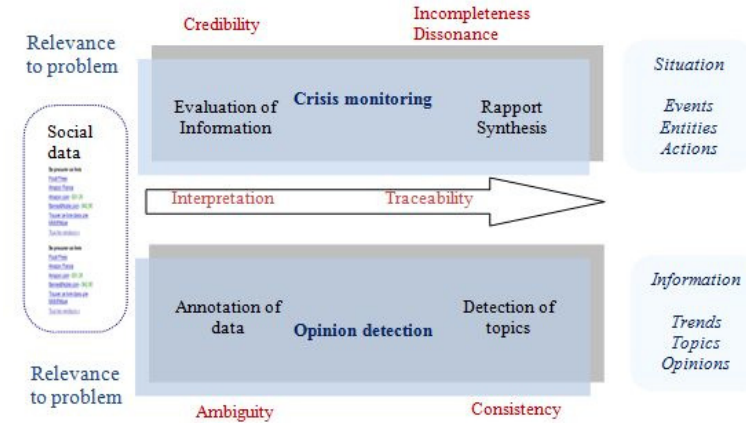

Fig. 4. URREF criteria for uncertainty analysis

By using the URREF ontology, uncertainty of social media analysis in two different contexts is described by a set of common criteria. Nevertheless, as shown in fig. 4, those criteria are to be estimated at different processing steps for each use case. 
The use of URREF criteria offers a common ground to assess uncertainties, once we identify how they enter into different processes and how data seta are processed and transformed by specific algorithms.

\section{CONCLUSiOn AND FUTURE WORK}

This paper addresses the exploitation of social data and discusses uncertainty criteria affecting the overall process from data collection interpretation of results. Uncertainty assessment is suitable for systems using data of questionable quality and there is a need to continuously update the quality of items in the system. As such, technical approaches for social data analysis have currently limited capabilities to explain the outcome. URREF criteria offer a unified basis to analyze inaccuracies affecting social data analysis during different phases: data gathering, enrichment and processing.

To overcome limitations of current approaches for social data analysis, a mapping to URREF uncertainty criteria is used. The mapping highlights when uncertainties are added into the system, which partial results and affected and facilitates the interpretation of results by adding additional information as to why results are to be trusted or not. Uncertainties can be made explicit not only for static elements of the model, such as sources or items, but also for the dynamic processes of combining items with one another. Moreover, a critical assessment is performed in order compare uncertainties arising in each use case.

Future work will address the edition of metrics for URREF criteria and the assessment of uncertainty metrics for different data sets and specific configurations within each use case.

\section{Acknowledgement}

Results reported in this document have been produced in the context of the ETUR working group. The authors are grateful to reviewers for their useful remarks.

\section{REFERENCES}

[1] P. Barberá and Z. C. Steinert-Threlkeld, "Social media as data generators," 2019.

[2] A. Sheth, P. Anantharam, and C. Henson, "Physical-cyber-social computing: An early 21 st century approach," IEEE Intelligent Systems, no. 1, pp. 78-82, 2013.

[3] M. J. Mazarr, A. Casey, A. Demus, S. W. Harold, L. J. Matthews, N. Beauchamp-Mustafaga, and J. Sladden, "Hostile social manipulation present realities and emerging trends," RAND Corporation Santa Monica United States, Tech. Rep., 2019.

[4] P. Wang, R. Angarita, and I. Renna, "Is this the era of misinformation yet: combining social bots and fake news to deceive the masses," in Companion Proceedings of the The Web Conference 2018. International World Wide Web Conferences Steering Committee, 2018, pp. $1557-$ 1561.

[5] E. Ferrara, "Contagion dynamics of extremist propaganda in social networks," Information Sciences, vol. 418, pp. 1-12, 2017.

[6] H. Alvari, S. Sarkar, and P. Shakarian, "Detection of violent extremists in social media," arXiv preprint arXiv:1902.01577, 2019.

[7] S. Kannangara, "Mining twitter for fine-grained political opinion polarity classification, ideology detection and sarcasm detection," in Proceedings of the Eleventh ACM International Conference on Web Search and Data Mining, 2018, pp. 751-752.

[8] T. Isbister, M. Sahlgren, L. Kaati, M. Obaidi, and N. Akrami, "Monitoring targeted hate in online environments," arXiv preprint arXiv:1803.04757, 2018.
[9] M. Hasan, M. A. Orgun, and R. Schwitter, "A survey on real-time event detection from the twitter data stream," Journal of Information Science, vol. 44, no. 4, pp. 443-463, 2018.

[10] Y. Wei and L. Singh, "Using network flows to identify users sharing extremist content on social media," in Pacific-Asia Conference on Knowledge Discovery and Data Mining. Springer, 2017, pp. 330-342.

[11] D. O'Callaghan, D. Greene, M. Conway, J. Carthy, and P. Cunningham, "Uncovering the wider structure of extreme right communities spanning popular online networks," in Proceedings of the 5th Annual ACM Web Science Conference, 2013, pp. 276-285.

[12] J. M. Berger and B. Strathearn, Who Matters Online: Measuring Influence, Evaluating Content and Countering Violent Exremism in Online Social Networks. International Centre for the Study of Radicalisation and Political Violence, 2013.

[13] R. Lara-Cabrera, A. Gonzalez-Pardo, M. Barhamgi, and D. Camacho, "Extracting radicalisation behavioural patterns from social network data," in 2017 28th International Workshop on Database and Expert Systems Applications (DEXA). IEEE, 2017, pp. 6-10.

[14] F. Hemmatian and M. K. Sohrabi, "A survey on classification techniques for opinion mining and sentiment analysis," Artificial Intelligence Review, pp. 1-51, 2017.

[15] J. Deriu, A. Lucchi, V. De Luca, A. Severyn, S. Müller, M. Cieliebak, T. Hofmann, and M. Jaggi, "Leveraging large amounts of weakly supervised data for multi-language sentiment classification," in Proceedings of the 26th international conference on world wide web, 2017, pp. 10451052 .

[16] L. Chen, J. Martineau, D. Cheng, and A. Sheth, "Clustering for simultaneous extraction of aspects and features from reviews," in Proceedings of the 2016 Conference of the North American Chapter of the Association for Computational Linguistics: Human Language Technologies, 2016, pp. 789-799.

[17] M. Thelwall and B. Cugelman, "Monitoring twitter strategies to discover resonating topics: The case of the undp," 2017.

[18] M. d. P. Salas-Zárate, R. Valencia-García, A. Ruiz-Martínez, and R. Colomo-Palacios, "Feature-based opinion mining in financial news: an ontology-driven approach," Journal of Information Science, vol. 43, no. 4, pp. 458-479, 2017.

[19] Z. Li, Y. Zhang, Y. Wei, Y. Wu, and Q. Yang, "End-to-end adversarial memory network for cross-domain sentiment classification." in IJCAI, 2017, pp. 2237-2243.

[20] F. H. Khan, U. Qamar, and S. Bashir, "Sentimi: Introducing point-wise mutual information with sentiwordnet to improve sentiment polarity detection," Applied Soft Computing, vol. 39, pp. 140-153, 2016.

[21] A. Agarwal, V. Sharma, G. Sikka, and R. Dhir, "Opinion mining of news headlines using sentiwordnet," in 2016 Symposium on Colossal Data Analysis and Networking (CDAN). IEEE, 2016, pp. 1-5.

[22] A. Esuli, "The user feedback on sentiwordnet," arXiv preprint arXiv:1306.1343, 2013.

[23] A. Schoene and G. de Mel, "Pooling tweets by fine-grained emotions to uncover topic trends in social media."

[24] P. Vijayaraghavan, S. Vosoughi, and D. Roy, "Automatic detection and categorization of election-related tweets," in Tenth International AAAI Conference on Web and Social Media, 2016.

[25] P. R. White, "Appraisal theory," The international encyclopedia of language and social interaction, pp. 1-7, 2015.

[26] D. L. McGuinness, F. Van Harmelen et al., "Owl web ontology language overview," W3C recommendation, vol. 10, no. 10, p. 2004, 2004.

[27] C. Laudy, "Hidden relationships discovery through high-level information fusion," in 2015 18th International Conference on Information Fusion (Fusion). IEEE, 2015, pp. 916-923.

[28] S. Fossier, C. Laudy, and F. Pichon, "Managing uncertainty in conceptual graph-based soft information fusion," in Proceedings of the 16th International Conference on Information Fusion. IEEE, 2013, pp. 930-937.

[29] G. Weimann, "Going dark: Terrorism on the dark web," Studies in Conflict \& Terrorism, vol. 39, no. 3, pp. 195-206, 2016.

[30] P. de Villiers, G. Pavlin, P. Costa, A.-L. Jousselme, K. Laskey, V. Dragos, and E. Blasch, "Subjects under evaluation with the urref ontology," in Information Fusion (Fusion), 2017 20th International Conference on. IEEE, 2017, pp. 1-8.

[31] J. de Villiers, R. Focke, G. Pavlin, A. Jousselme, V. Dragos, K. Laskey, P. Costa, and E. Blasch, "Evaluation metrics for the practical application of urref ontology: An illustration on data criteria," in Information Fusion (Fusion), 2017 20th International Conference on. IEEE, 2017, pp. 1-8. 
[32] V. Dragos, "An ontological analysis of uncertainty in soft data," in Information Fusion (FUSION), 2013 16th International Conference on. IEEE, 2013, pp. 1566-1573.

[33] E. Blasch, K. B. Laskey, A.-L. Jousselme, V. Dragos, P. C. Costa, and J. Dezert, "Urref reliability versus credibility in information fusion (stanag 2511)," in Information Fusion (FUSION), 2013 16th International Conference on. IEEE, 2013, pp. 1600-1607.

[34] E. Blasch, A. Jøsang, J. Dezert, P. C. Costa, and A.-L. Jousselme, "Urref self-confidence in information fusion trust," in Information Fusion (FUSION), 2014 17th International Conference on. IEEE, 2014, pp. $1-8$.

[35] E. Blasch and A. Aved, "Urref for veracity assessment in query-based information fusion systems," in Information Fusion (Fusion), 2015 18th International Conference on. IEEE, 2015, pp. 58-65.

[36] J. P. de Villiers, K. Laskey, A.-L. Jousselme, E. Blasch, A. de Waal, G. Pavlin, and P. Costa, "Uncertainty representation, quantification and evaluation for data and information fusion," in Information Fusion (Fusion), 2015 18th International Conference on. IEEE, 2015, pp. $50-57$.

[37] V. Dragos, J. Ziegler, and J. P. De Villiers, "Application of urref criteria to assess knowledge representation in cyber threat models," in $201821 \mathrm{st}$ International Conference on Information Fusion (FUSION). IEEE, 2018, pp. 664-671.

[38] J. P. de Villiers, A.-L. Jousselme, A. de Waal, G. Pavlin, K. Laskey, E. Blasch, and P. Costa, "Uncertainty evaluation of data and information fusion within the context of the decision loop," in Information Fusion (FUSION), 2016 19th International Conference on. IEEE, 2016, pp. 766-773.

[39] V. Dragos, X. Lerouvreur, and S. Gatepaille, "A critical assessment of two methods for heterogeneous information fusion," in Information Fusion (Fusion), 2015 18th International Conference on. IEEE, 2015, pp. 42-49.

[40] H. Koen, J. P. de Villiers, G. Pavlin, A. de Waal, P. de Oude, and F. Mignet, "A framework for inferring predictive distributions of rhino poaching events through causal modelling," in Information Fusion (FUSION), 2014 17th International Conference on. IEEE, 2014, pp. $1-7$.

[41] E. Blasch, P. C. Costa, K. B. Laskey, H. Ling, and G. Chen, "The urref ontology for semantic wide area motion imagery exploitation," in Aerospace and Electronics Conference (NAECON), 2012 IEEE National. IEEE, 2012, pp. 228-235. 